\section{Response to "Factors associated with long-term impact on informal caregivers during Alzheimer's disease dementia progression: 36-month results from GERAS"}

We read with interest the recent paper entitled "Factors associated with long-term impact on informal caregivers during Alzheimer's disease dementia progression: 36-month results from GERAS" by Reed et al. (2019). The authors concluded that long-term informal caregiver burden is driven by declining functional abilities and increased behavioral symptoms in patients with Alzheimer's disease in the community.

Longitudinal studies using large sample sizes which focused on the burden of caregivers of persons with dementia are few. Previous analysis of longitudinal data assessing the burden of caregivers of persons with dementia over 12 months (Kajiwara et al., 2018) found no significant differences in caregiver burden, positive caregiving appraisal, and behavioral and psychological symptoms of dementia (BPSD). Moreover, other studies reported that BPSD was associated with caregiver burden (Kajiwara et al., 2015; van der Linde et al., 2012). This study demonstrated worsening behavioral symptoms in patients with Alzheimer's disease. Therefore, caregiver burden may be positively associated with BPSD in this study and this information is important to consider.

It is noteworthy that Reed et al., (2019) reported lower increases in caregiver burden over 36 months when the participants lived in Germany. A previous study reported differences in caregiver burden by country (Matsushita et al., 2016). Other research in Sweden and the United States have reported that caregiver burden tends to decline in longitudinal studies (Gaugler et al., 2009; Grafström \& Winblad, 1995). Therefore, it may be useful to consider the relationship between cultural background and caregiver burden in each country, as there may be differences in caregiving roles.

Undoubtedly, it is important to focus on longterm impacts of caregiver burden, and to use large sample sizes in longitudinal designs. The study conducted by Reed et al. (2019) provided useful data in this regard. We believe that longitudinal studies emphasizing caregiver burden, functional abilities and behavioral symptoms will be beneficial in understanding the burdens of caregivers of persons with dementia.

\section{Conflict of interest}

None.

\section{Description of authors' roles}

$\mathrm{KK}, \mathrm{JK}, \mathrm{HN}, \mathrm{YO}$ and $\mathrm{MK}$ made substantial contributions to study conception and design. KK and JK were involved in drafting the manuscript or revising it. All authors contributed to and approved all drafts.

\section{References}

Gaugler, J.E. et al. (2009). Predictors of change in caregiver burden and depressive symptoms following nursing home admission. Psychology and Aging, 24, 385-396.

Grafström, M. and Winblad, B. (1995). Family burden in the care of the demented and nondemented elderly: a longitudinal study. Alzheimer Disease \& Associated Disorders, 9, 78-86.

Kajiwara, K., et al. (2015). Positive appraisal of in-home family caregivers of dementia patients as an influence on the continuation of caregiving. Psychogeriatrics, 15, 26-31.

Kajiwara, K., Noto, H. and Yamanaka, M. (2018). Changes in caregiving appraisal among family caregivers of persons with dementia: a longitudinal study over 12 months. Psychogeriatrics, 18, 460-467.

Matsushita, M., et al. (2016). Cross-cultural study of caregiver burden for Alzheimer's disease in Japan and Taiwan: Result from Dementia Research in Kumamoto and Tainan (DeReKaT). International Psychogeriatrics, 28, 1125-1132.

Reed, C., et al. (2019). Factors associated with long-term impact on informal caregivers during Alzheimer's disease dementia progression: 36-month results from GERAS. International Psychogeriatrics, doi: 10.1017/ S1041610219000425.

van der Linde, R.M., et al. (2012). Systematic reviews on behavioural and psychological symptoms in the older or demented population. Alzheimer's Research and Therapy, 4,28 .

Kohei Kajiwara, ${ }^{1}$ (i) Jun Kako, ${ }^{1}$ Hiroko Noto, ${ }^{2}$ YASUfumi Oosono $^{3}$ and Masamitsu Kobayashi ${ }^{4}$

${ }^{1}$ Division of Nursing Science, Graduate School of Biomedical and Health Sciences, Hiroshima University, Minami-ku, Hiroshima, 734-8553, Japan 
${ }^{2}$ Department of Health Science, Graduate School of Medical Sciences, Kyushu University, Fukuoka, Japan ${ }^{3}$ Community Health Nursing, Ministry of Defense National Defense Medical College, Saitama, Tokorozawa, Japan

${ }^{4}$ Faculty of Nursing, National Defense Medical College, Tokorozawa, Japan
Correspondence should be addressed to: Kohei Kajiwara, Division of Nursing Science, Graduate School of Biomedical and Health Sciences, Hiroshima University, 1-2-3, Kasumi, Minami-ku, Hiroshima 734-8553, Japan. E-mail: kkaji@hiroshima-u.ac.jp 